\section{Facharztprüfung zur Erlangung des Facharzttitels FMH für Kinderchirurgie}

Aufgrund des Weiterbildungsprogrammes und des Beschlusses des Zentralvorstandes ist das Bestehen der Facharztprüfung Voraussetzung zur Erlangung des Facharzttitels FMH für Kinderchirurgie. Das Bestehen des Basisexamens Chirurgie ist obligatorisch. Kandidaten, welche die Anforderungen für die Erlangung des Facharztes FMH für Kinderchirurgie erfüllen oder die im letzten Weiterbildungsjahr stehen, melden sich bei der Präsidentin der Weiterbildungskommission, Frau Prof. Dr. med. Claude Le Coultre.
Datum der mündlichen Prüfung: Freitag, 16. November 2001

Ort: Kinderspital, Genf

Prüfungsgebühr: Die SGKC erhebt eine Prüfungsgebühr von Fr. 500.-.

Anmeldung (unter Beilage des Curriculum vitae): Prof. Dr. med. Claude le Coultre, Präsidentin der Weiterbildungskommission, Hôpital des Enfants, Clinique et Policlinique de chirurgie pédiatrique, 6, rue Willy-Donzé, 1211 Genève 14, Tel. 022 38246 62, Fax 02238246 66, E-mail: claude. lecoultre@hcuge.ch

Anmeldefrist: 16. September 2001

\section{Examen de spécialiste en vue de l'obtention du titre de spécialiste FMH en chirurgie pédiatrique}

Conformément au programme de formation postgraduée et à la décision du Comité central de la FMH, la réussite de l'examen de spécialiste est une condition requise pour les candidats au titre de FMH en chirurgie pédiatrique. La réussite à l'examen des connaissances de base en chirurgie générale est obligatoire. Les candidats remplissant les conditions ou se trouvant en dernière année de formation doivent s'annoncer par écrit auprès de la Présidente de la formation postgraduée, Madame le Professeur Claude le Coultre.
Date de l'examen oral: Vendredi, 16 novembre 2001 Lieu d'examen: Hôpital des Enfants, Genève

Taxe d'examen: La SSCI prélève une participation aux frais de Fr. 500.--

Inscription (accompagnée du curriculum vitae): Prof. Claude le Coultre, Présidente de la formation postgraduée, Hôpital des Enfants, Clinique et Policlinique de chirurgie pédiatrique, 6 , rue WillyDonzé, 1211 Genève 14, tél. 02238246 62, fax 02238246 66, e-mail: claude.lecoultre@hcuge.ch

Delai de l'inscription: 16 septembre 2001 